\title{
Letter
}

\section{How to reduce the risk of failing to reach recruitment targets: lessons learnt from a pump pilot trial}

Diabet. Med. 29, 1600-1601 (2012)

Large-scale multi-centre randomized controlled trials (RCTs) are regarded as the gold standard in rigorous, robust clinical research. Rates of participant recruitment, however, are often challenging and can have major impact on the planning, execution and funding of trials. Failure to recruit sufficient numbers of participants, or extended delays in recruitment, can threaten the trial's success and resources, in terms of both time and money. Trials frequently fail to recruit to target. In 2007, Campbell and colleagues reported that more than $80 \%$ of all trials fall short of their accrual goals, potentially seriously compromising the validity of the findings [1]. UK publicly funded (Medical Research Council and Health Technology Assessment) trials are no more successful. Between 1 January 1994 and 31 December 2002, only 35/114 (31\%) of trials recruited successfully (i.e. equal to or above original recruitment target).

Several common factors contribute to poor recruitment, including overestimation of the pool of available and suitable participants, underestimation of the time required to recruit participants across multiple centres, willingness of both patients and clinicians to participate, and lack of contingency plans when recruiting fails [2]. We conducted a survey of people who declined to take part in the pilot study to inform development of the Relative Effectiveness of Pumps Over Structured Education trial (REPOSE) trial. The aims were to explore the reasons for non-participation in the pump pilot study, understanding of insulin pump therapy and to use this information to inform recruitment processes in the multi-centre REPOSE randomized controlled trial.

Ethics approval was received from the National Health Service (NHS) Central Office for Research Ethics Committees. Participants were selected from those who had completed a screening questionnaire asking them about their reasons for not wishing to participate in the pump pilot and who had expressed a willingness to be interviewed to discuss their non-participation to the trial. Eight participants were selected covering a representative cross section of reasons for non-participation. Interviews ranged from 14 to $34 \mathrm{~min}$ (median $24.5 \mathrm{~min}$ ). The age range of participants was 22-59 years (median 40 years). Duration of diabetes was $10-33$ years (mean 18.8 years). All participants were using multiple daily injection (MDI) insulin regimens, and five had attended a Dose Adjustment for Normal Eating (DAFNE) structured education course within the previous 12 months. Reasons for non-participation included lack of time, inconvenience, lack of interest in insulin pump and satisfaction with current treatment.

Overall there was poor understanding of insulin pump therapy, with three quarters of participants reporting very little knowledge of either how the pump works or what it looks like. Mixed and sometimes contradictory suggestions were proposed for how the research team could make it easier to participate. A desire for as much information as possible was countered with a preference for less detail, preferring something 'short and succinct'. Availability face-to-face vs. availability via phone/ email of healthcare professionals to discuss the research project in more detail highlight the need for as broad a range as possible for patient contact, as well as provision of evening appointments around work prior to having to sign up. This personal contact preference is consistent with previous literature [3]. Perhaps the most effective approach, offering practicality and personal contact would be the use of recruitment meetings [4]. Having an insulin pump available in clinic for potential participants to see could improve understanding and remove some potential anxieties about constant attachment and cannula insertion. Myths and misinformation about insulin pumps continue to exist, and lack of knowledge about the appearance or functions of insulin pump therapy also deter some people.

The potential to offer participants incentives, such as shop vouchers, to respond to questionnaires (baseline and followup) was not raised in this brief study, but they may be useful when considering how to maximize recruitment. A recent Cochrane review reported that 'researchers may be able to double the odds of response by offering participants payment for completion of questionnaires' [3]. This could be explored in greater detail as an extension to existing research precedents; for example, cash incentives to promote smoking cessation. Maintaining participation throughout the REPOSE trial and 2-year follow-up is important, as the power of the study is dependent on at least $80 \%$ of participants being retained. A more detailed explanation of pumps and their potential advantages and disadvantages is required prior to inviting people to participate in large multi-centre clinical trials.

In conclusion, the commonest reasons for non-participation were a lack of understanding and knowledge of pump therapy and satisfaction with current treatment. Targeted education in providing clear explanations of the technology being evaluated, together with its potential advantages and disadvantages, is an important part of recruitment in large trials, and potential recruits should be offered clear information in a way that suits their needs. 
K. D. Barnard, M. J. Campbell, C. Emery,

A. J. Young and S. Heller for the DAFNE UK Study Group DAFNE UK Study Group, Sheffield, UK

\section{Funding sources}

None.

\section{Competing interests}

Nothing to declare.

\section{Acknowledgement}

This article presents independent research commissioned by the National Institute for Health Research (NIHR) under its Programme Grants for Applied Research scheme (RP-PG-
0606-1184). The views expressed in this publication are those of the author(s) and not necessarily those of the NHS, the NIHR or the Department of Health.

\section{References}

1 Campbell MK, Snowdon C, Francis D, Elbourne D, McDonald AM, Knight $\mathrm{R}$ et al. Recruitment to randomised trials: strategies for trial enrolment and participation study. The STEPS study. Health Technol Assess 2007; 11: 48.

2 Klabunde CN, Springer BC, Butler B, White MS, Atkins J. Factors influencing enrolment in clinical trials for cancer treatment. South Med J 1999; 92: 1189-1193.

3 Edwards PJ, Roberts I, Clarke MJ, DiGuiseppi C, Wentz R, Kwan I et al. Methods to increase response to postal and electronic questionnaires. Cochrane Database Syst Rev 2009; 3: MR000008.

4 DAFNE Study Group. Training in flexible, intensive insulin management to enable dietary freedom in people with type 1 diabetes: dose adjustment for normal eating (DAFNE) randomised controlled trial. Br Med J 2002; 325: 746-750. 